\title{
COMPACT STELLAR BINARY ASSEMBLY IN THE FIRST NUCLEAR STAR CLUSTERS AND $r$-PROCESS SYNTHESIS IN THE EARLY UNIVERSE
}

\author{
Enrico Ramirez-Ruiz ${ }^{1,2,3}$, Michele Trenti ${ }^{4,5}$, Morgan MacLeod ${ }^{1}$, Luke F. Roberts ${ }^{6,8}$, \\ William H. LeE ${ }^{7}$, and Martha I. Saladino-Rosas ${ }^{7}$ \\ ${ }^{1}$ Department of Astronomy and Astrophysics, University of California, Santa Cruz, CA 95064, USA \\ ${ }^{2}$ Radcliffe Institute for Advanced Study, Harvard University, Cambridge, MA 02138, USA \\ ${ }^{3}$ Niels Bohr Institute, University of Copenhagen, DK-2100 Copenhagen, Denmark \\ ${ }^{4}$ Kavli Institute for Cosmology and Institute of Astronomy, University of Cambridge, Madingley Road, Cambridge CB3 OHA, UK \\ ${ }^{5}$ School of Physics, University of Melbourne, VIC 3010, Australia \\ ${ }^{6}$ TAPIR, California Institute of Technology, Pasadena, California 91125, USA \\ ${ }^{7}$ Instituto de Astronomía, Universidad Nacional Autónoma de México, México DF 04510, México \\ Received 2014 October 13; accepted 2015 March 16; published 2015 April 1
}

\begin{abstract}
Investigations of elemental abundances in the ancient and most metal deficient stars are extremely important because they serve as tests of variable nucleosynthesis pathways and can provide critical inferences of the type of stars that lived and died before them. The presence of $r$-process elements in a handful of carbon-enhanced metalpoor (CEMP-r) stars, which are assumed to be closely connected to the chemical yield from the first stars, is hard to reconcile with standard neutron star mergers. Here we show that the production rate of dynamically assembled compact binaries in high- $z$ nuclear star clusters can attain a sufficient high value to be a potential viable source of heavy $r$-process material in CEMP- $r$ stars. The predicted frequency of such events in the early Galaxy, much lower than the frequency of Type II supernovae but with significantly higher mass ejected per event, can naturally lead to a high level of scatter of Eu as observed in CEMP- $r$ stars.
\end{abstract}

Key words: early universe - galaxies: evolution - galaxies: high-redshift - stars: abundances

\section{INTRODUCTION}

The oldest persisting stars in the galactic halo serve as laboratories for studies of neutron-capture element synthesis in the early universe (Sneden et al. 2008). Their chemical compositions provide evidence about the identities of the first generations of stars that lived and died before them (Truran et al. 2002; Matteucci et al. 2014). $r$-process elements are commonly observed in stars with metallicities $[\mathrm{Fe} / \mathrm{H}] \lesssim-3$, indicating that their progenitors must have been relatively swiftly evolving. Of particular significance is the rich $r$-process element composition found in some carbon-enhanced metalpoor (CEMP-r) stars (e.g., CS 22892-052; Sneden et al. 2003; Masseron et al. 2010). The presence of carbon in these stars has been closely connected to the chemical yield from the first stars 9 (Umeda \& Nomoto 2003; Cooke \& Madau 2014) while the $r$-process must either come from the same first stars or from a source that acts on a timescale shorter than the time required to form a second generation of stars.

The neutrino driven wind in Type II supernovae ( $\mathrm{SNe}$ ) has long been considered a likely site for $r$-process synthesis, based on both the physical conditions found in early simulations (Woosley et al. 1994) and chemical evolution considerations in the early universe (Argast et al. 2004; Cowan \& Thielemann 2004). Later work has shown that it is difficult to get conditions in the wind that produce the $r$-process (e.g., Takahashi et al. 1994; Qian \& Woosley 1996). Neutron star (NS) mergers offer a robust alternative to Type II SNe (Lattimer \& Schramm 1974; Freiburghaus et al. 1999). The $r$ process nuclei are robustly synthesized in the matter ejected in

\footnotetext{
${ }^{8}$ NASA Einstein Fellow.

${ }^{9}$ However, it is still debated whether these enhancements are related to the star's birth composition, or if its atmosphere was subsequently polluted by a binary companion (e.g., Starkenburg et al. 2014).
}

such mergers (Metzger et al. 2010; Roberts et al. 2011; Bauswein et al. 2013; Grossman et al. 2014) and their predicted enrichment history is in agreement with abundance patterns observed in galactic halo stars (Shen et al. 2014; van de Voort et al. 2014). However, because evolved NS binaries are expected to merge hundreds to thousands of millions of years after birth (Fryer et al. 1999; Kalogera et al. 2001; Belczynski et al. 2006; Behroozi et al. 2014), a shorter-lived merging channel might be required in order to explain $r$-process enrichment in CEMP- $r$ stars.

In this Letter, we explore one such channel by investigating whether or not sufficient $r$-process material can be synthesized in the first star clusters formed within the first $500 \mathrm{Myr}$ after Big Bang via dynamically assembled merging compact binaries (Lee 1993; Lee et al. 2010; Samsing et al. 2014). To answer this question, we first estimate the formation rate of nuclear star clusters in the early universe as well as their structural properties, which we use to compute detailed estimates of the rate of compact object encounters within such systems (Section 2). From the stellar binary merger rate, we then derive the amount of $r$-process material assembled in the early universe using the typical mass production rate per event, which is addressed in detail for compact binary encounters in Section 3. Discussion and conclusions are presented in Section 4.

\section{COMPACT BINARY ASSEMBLY IN THE EARLY UNIVERSE}

\subsection{The Formation Rate and Characteristics of High-z Star Clusters}

The basic assumption of our modeling is that compact star clusters can be formed at the center of high- $z$ dark matter (DM) halos with a virial temperature $T_{\text {vir }} \sim(1-2) \times 10^{4} \mathrm{~K}$ and non- 


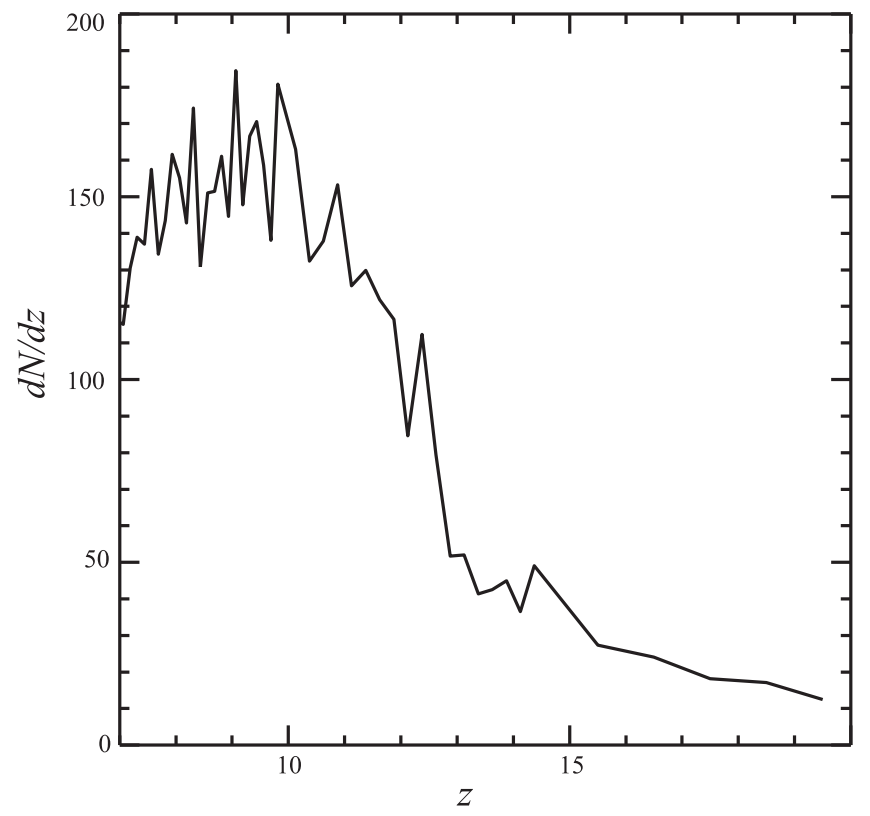

Figure 1. Formation rate for central star clusters as inferred from three Milky-Way-like halos from Trenti \& Shull (2010). Shown are the number of clusters per unit redshift that will be part of a Milky-Way-like halo at $z=0$, which are assumed to form at the center of dark matter halos with $T_{\mathrm{vir}} \gtrsim 10^{4} \mathrm{~K}$ and $Z \sim 10^{-4} Z_{\odot}$.

zero but very low metallicity $\log _{10}\left(Z / Z_{\odot}\right) \sim-4$. In fact, under these conditions disk fragmentation can be suppressed and gas efficiently funneled by the Toomre instability in the innermost few parsecs where it forms a star cluster (see Devecchi \& Volonteri 2009 for details).

To estimate the formation rate of early forming compact and high-density star clusters, we derive the formation rate of DM halos meeting the criteria above from the simulations of Trenti \& Shull (2010), which focus on early chemical enrichment of three Milky-Way-like halos with DM mass $M_{\mathrm{DM}}(z=0) \approx 10^{12} M_{\odot}$. The simulations have been carried out using $N_{\mathrm{DM}}=1024^{3} \mathrm{DM}$ particles in a comoving volume of $10^{3} \mathrm{Mpc}^{3}$, with DM mass resolution of $3.4 \times 10^{4} \mathrm{M}_{\odot}$. The DM only simulation is post-processed with the star formation and chemical enrichment model described in Trenti \& Shull (2010) and Trenti et al. (2009), which includes treatment of metal outflows and radiative feedback in the Lyman-Werner bands to regulate Population III formation (Trenti \& Stiavelli 2009).

Because, as shown in Section 2.2, the encounter rate in the core of the first star clusters declines significantly at redshifts $z<10$ (mainly due to a decrease in the central density), we focus only on systems formed before then, which are shown in Figure 1. By $z=10$, a total of $\left\langle N_{c}\right\rangle=515$ clusters are expected to be formed in a region that will collapse by $z=0$ in a Milky-Way-like halo. This number is higher by a factor of about two when compared to the formation rate of similar objects in a random region of the universe, owing to the enhanced DM halo formation rate induced by the over dense environment (see, e.g., Trenti \& Shull 2010).

Overall our simplified approach provides a robust estimate of the formation rate of halos capable of hosting dense star clusters. For example, feedback at $z>10$ is unlikely to affect gas in halos with $T_{\text {vir }}>10^{4} \mathrm{~K}$. This leaves the leading source of uncertainty in the details of star formation in such halos, which are very challenging to model from first principles (e.g.,
Wise et al. 2012). Thus, as a first characterization, we describe the internal properties of first stellar clusters at $z \approx 10$ following Devecchi \& Volonteri (2009). From Figure 4 in Devecchi \& Volonteri (2009) we derive the characteristic stellar mass $M_{\mathrm{tot}}$ and half-mass radius $\eta_{\mathrm{hm}}$ for the central star clusters as a function of their formation redshift. While the core density during initial stages of cluster evolution is fairly uncertain and dependent upon the initial conditions, as a basic estimate, based on direct N-body modeling experiments (Trenti et al. 2010), we assume a typical core to half mass radius ratio $r_{c} / r_{\mathrm{hm}}=0.1$ and a core to characteristic stellar mass ratio $M_{c} / M_{\mathrm{tot}}=0.04$, corresponding to a pre-core collapse cluster with a $W_{0}=8.5 \mathrm{King}$ (1966) profile. With the model (in virial equilibrium), we derive the central velocity dispersion from the cluster mass and radius:

$$
\sigma_{c}=18.81 \mathrm{~km} \mathrm{~s}^{-1}\left(\frac{M_{\mathrm{tot}}}{10^{5} M_{\odot}}\right)\left(\frac{1 \mathrm{pc}}{r_{\mathrm{hm}}}\right) .
$$

Having an estimate for the mass and velocity dispersion of a cluster (Figure 2), the number of NSs can be calculated by assuming the star clusters have a Kroupa (2001) IMF $\xi(m)=\xi_{0}\left(m / 0.5 M_{\odot}\right)^{\alpha(m)}$, with $m \in\left[m_{\min }: m_{\max }\right]$ and either a cut-off at $m_{\min }=0.2 M_{\odot}$ or at $m_{\min }=1 M_{\odot}$ (top-heavy IMF). $m_{\max }=100 M_{\odot}, \alpha(m)=-2.3$ for $m>0.5 M_{\odot}$ and $\alpha(m)=-1.3$ for $0.2 M_{\odot} \leqslant m \leqslant 0.5 M_{\odot}$. By assuming, for example, that NSs are produced in the mass range [8:25] $M_{\odot}$ (Hurley et al. 2000), the fraction of NSs is $f_{\mathrm{ns}}=9 \times 10^{-3}$ for the standard IMF and $f_{\mathrm{ns}}=5 \times 10^{-2}$ for the top heavy IMF.

Next, we estimate the fraction of these NSs that are retained in the cluster core. The core retention fraction is affected by NS natal kicks and binary and mass segregation. We assume that the progenitors of NSs are generally in binaries since massive stars are expected to form with high binarity (Krumholz et al. 2009), and use the model of Pfahl et al. (2002, Figure 12) to estimate the retention of NSs formed in clusters with different escape velocities. We consider a Maxwellian kick distribution, $\quad p(v)=\sqrt{2 / \pi} v^{2} / \sigma_{\text {kick }}^{3} e^{-v^{2} /\left(2 \sigma_{\text {kick }}^{2}\right)}, \quad$ with $\sigma_{\text {kick }}=100 \mathrm{~km} \mathrm{~s}^{-1}$ and $\sigma_{\text {kick }}=200 \mathrm{~km} \mathrm{~s}^{-1}$, which are the fastest kick distribution studied by Pfahl et al. (2002). Since that study concludes that even with their optimistic assumptions they might underestimate the actual retention fraction (Section 7 in Pfahl et al. 2002), our approach is fairly conservative. We assume a cluster escape speed of $v_{\mathrm{esc}}(0)=4.12 \sigma_{\mathrm{c}}$ as derived from the King density profile. In what follows we will assume that $50 \%$ of the retained NSs are unbound from their parent binary while $50 \%$ remain bound, as roughly found by Pfahl et al. (2002) for the range of cluster's escape velocities derived here. Mass segregation can enhance the number of NSs and NS progenitors in the core relative to the cluster mean. From N-body simulations with stellar evolution (M. MacLeod et al. 2015, in preparation), we see that mass segregation of the NS progenitors is efficient (core density increased by $\approx 4$ ), but the core density of NSs is increased only by $\approx 50 \%$, owing to the redistribution following natal kick. We thus apply this latter correction factor (that is $1.5 \times)$ to map the global to core retention fraction $\left(\zeta_{\text {ret }}\right)$, which is then shown in panel (d) of Figure 2. $\zeta_{\text {ret }} \sim 0.2$ for fast kicks $\left(\sigma_{\text {kick }}=200 \mathrm{~km} \mathrm{~s}^{-1}\right)$ at all redshifts, while for the distribution with slower kicks $\zeta_{\text {ret }} \sim 0.8$ at $z=14$, decreasing to $\zeta_{\text {ret }} \sim 0.55$ by $z \sim 10$. 

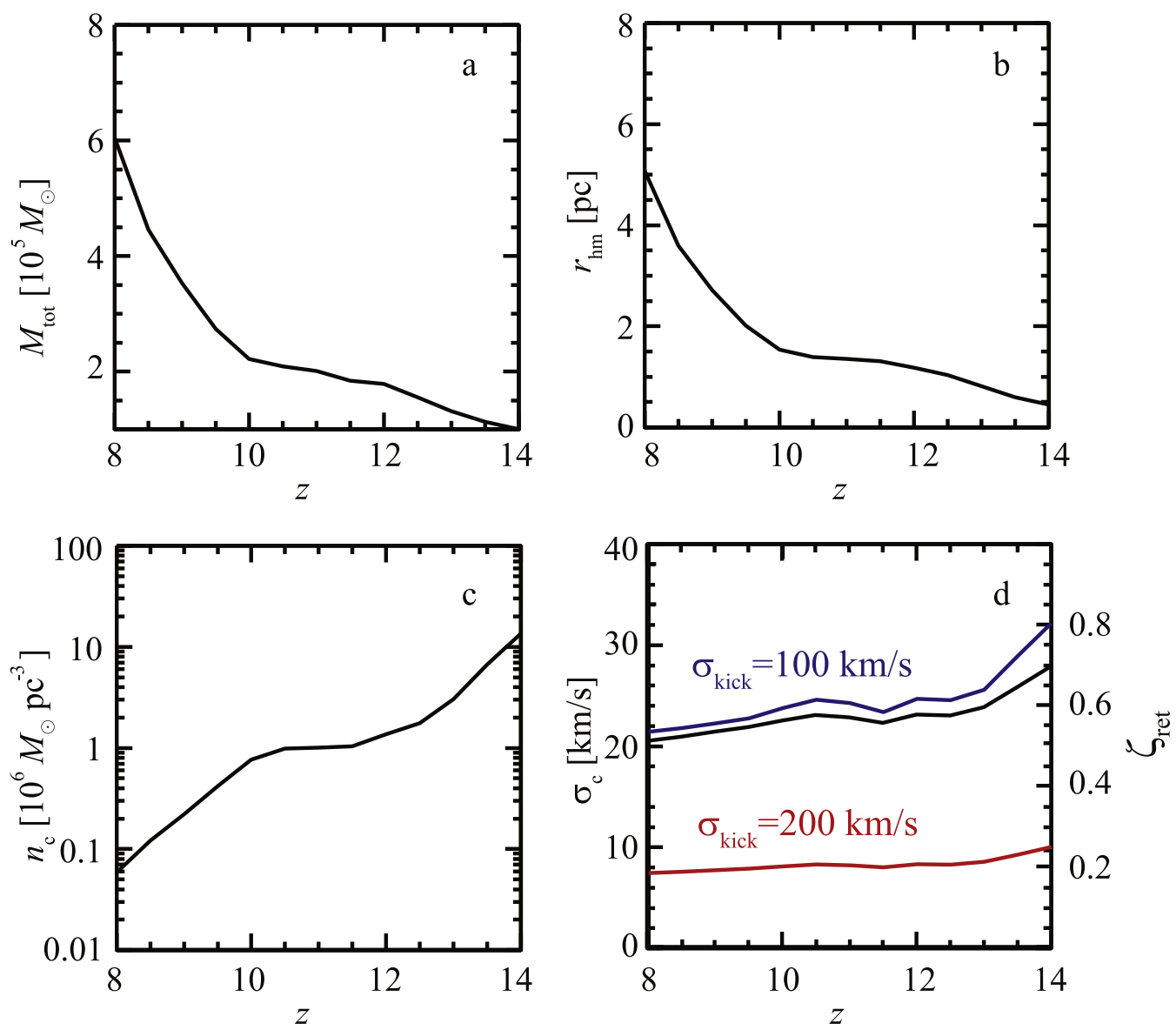

Figure 2. Structural properties of high- $z$ stellar clusters as a function of the redshift of formation, as predicted by the Devecchi \& Volonteri (2009) model for mass and radius and combined with the typical structural parameters from Trenti et al. (2010) to infer core properties. Plotted are the characteristic stellar mass $M_{\text {tot }}$ (panel a), half-mass radius $r_{\mathrm{hm}}$ (panel b), core stellar density $n_{c}$ (panel c) and core velocity dispersion $q_{c}$ (panel d; black line). Panel (d) also shows the core retention fraction $\zeta_{\text {ret }}$ for natal kicks with Maxwellian distribution and dispersion $\sigma_{\text {kick }}=100 \mathrm{~km} \mathrm{~s}^{-1}$ (blue line) and at $\sigma_{\text {kick }}=200 \mathrm{~km} \mathrm{~s}^{-1}$ (red line).

\subsection{NS Binary Assembly Rates}

To calculate the encounter rate within the cluster we assume NSs are distributed homogeneously within the core, with fractional number $f_{\mathrm{ns}}$, total number density $n_{c}$, and binary fraction $b_{\mathrm{ns}}$. We further assume that the stars follow a Maxwellian velocity distribution function with dispersion $\sigma_{c}$. We calculate a fiducial encounter rate $\xi_{\text {ns }}$ for individual clusters provided a distance of closest approach $R_{\min }$ (e.g., Lee et al. 2010)

$$
\begin{aligned}
\xi_{\mathrm{ns}}= & 3 \times 10^{-3} \mathrm{Gyr}^{-1} f_{\mathrm{ns}}^{2}\left(\frac{n_{c}}{10^{6} \mathrm{pc}^{-3}}\right)^{2}\left(\frac{r_{c}}{0.1 \mathrm{pc}}\right)^{3} \\
& \times\left(\frac{\sigma_{c}}{20 \mathrm{~km} \mathrm{~s}^{-1}}\right)^{-1}\left(\frac{M_{\mathrm{ns}}}{1.4 M_{\odot}}\right)\left(\frac{R_{\min }}{10 \mathrm{~km}}\right) .
\end{aligned}
$$

To estimate the rate of single-single NS encounters, we multiply by the single NS fraction squared, $1 / 2\left(1-b_{\mathrm{ns}}\right)^{2}$, where the factor of $1 / 2$ avoids double counting pairwise encounters. To estimate binary-single encounters, we must include a factor $b_{\mathrm{ns}}\left(1-b_{\mathrm{ns}}\right)$. To form a binary by transferring orbital energy to internal stellar oscillations, we adopt the cross section for tidal capture formalism described in Lee \& Ostriker (1986) and Kim \& Lee (1999), which in the case of
NSs gives

$$
R_{\mathrm{min}}^{\mathrm{tidal}}=13.37 R_{\mathrm{ns}}
$$

(Lee et al. 2010). To compute the cross section for binary formation by GW radiation, we follow Lee (1993). That is:

$$
R_{\min }^{\mathrm{gw}}=1458\left(\frac{\sigma_{c}}{20 \mathrm{~km} \mathrm{~s}^{-1}}\right)^{-4 / 7} \mathrm{~km} .
$$

This is significantly larger than $R_{\min }^{\text {tidal }}=160\left(R_{\mathrm{ns}} / 12 \mathrm{~km}\right) \mathrm{km}$. Although, as we show in Section 3, tidal capture encounters eject significantly more $r$-process material.

Finally, we consider encounters between one binary containing a NS with a single NS leading to an exchange, and subsequent formation of a NS-NS pair. Using exchange cross sections from Heggie et al. (1996), the cross section for a 1.4 $M_{\odot}$ NS to exchange into a binary given an equal mass companion is $\sigma_{\mathrm{ex}}=32.2 \mathrm{AU}^{2}(a / \mathrm{AU})\left(v / 20 \mathrm{~km} \mathrm{~s}^{-1}\right)^{-2}$. If the eccentricity is sufficiently high, the pair will merge on a short timescale (Samsing et al. 2014). To calculate the cross section for the formation of NS binaries with lifetime less than $1 \mathrm{Gyr}$, we use the lifetime estimate $t_{\text {life }} \approx 2.9 \times 10^{17}\left(1-e^{2}\right)^{7 / 2} \mathrm{yr}$ and assume thermal distribution of eccentricity, $p(e)=2 e$, following the exchange (Samsing et al. 2014). Thus, for any binary semimajor axis, $a$, there is a critical eccentricity $e_{\text {crit }}$ above which newly formed binaries will merge in less than 
1 Gyr. The fraction of binaries with $e>e_{\text {crit }}$ is thus $1-e_{\text {crit }}^{2}$ and scales as $a^{-8 / 7}$. The resultant cross section scales weakly with binary semimajor axis, $a^{-1 / 7}$ (e.g., Samsing et al. 2014, Figure 15). For a representative initial binary population with a binary semimajor axis distribution with $p(a) \propto a^{-1}$ and $a \in\left[10 R_{\odot}: 1 \mathrm{AU}\right]$, the integrated cross-section can be expressed as

$$
R_{\text {min }}^{3-\text { body }}=1.6 \times 10^{6} \mathrm{~km},
$$

in Equation (2). ${ }^{10}$

Equipped with $R_{\min }^{\text {tidal }}, R_{\min }^{\mathrm{gw}}$, and $R_{\min }^{3-\text { body }}$, we can calculate the binary assembly rate $\xi_{\text {ns }}$ for individual clusters (Equation (2)) as a function of their formation redshift, which, as shown in Figure 2, sets their structural properties. The rates for tidal, GW captured, and dynamically formed binaries within individual clusters are given in panel (a) of Figure 3, where we assume $b_{\mathrm{ns}}=0.5$. As expected, tidal encounters are very rare and the rate is dominated by binary-single interactions, which have been proposed as viable merger channel by several studies (Sigurdsson \& Phinney 1993; Grindlay et al. 2006; Samsing et al. 2014). In this work we ignore binary-binary encounters and their exchange products, which will represent, at most, a contribution of order unity to the binary-single encounter rate.

An estimate of the integrated encounter rate $\eta_{\text {ns }}$ over all the Milky Way central star clusters present at high $z$ can then be made under the assumption that each cluster contributes to the encounter rate from its redshift of formation until its dissolution. Conservatively, we assume that dissolution happens $0.5 \mathrm{Gyr}$ after cluster formation, although some clusters may well survive for longer and therefore provide additional contribution to the encounter rate. ${ }^{11}$ Our estimate of $\eta_{\text {ns }}$ for dynamically formed binaries as a function of $z$ is shown in panel (b) of Figure 3. From this panel it is possible to compute the total number of mergers (which will follow within $1 \mathrm{Gyr}$ after binary capture) in the Milky Way by integrating the rate over time. In panel (c) of Figure 3 we also plot for clarity the fraction of high- $z$ star clusters that experienced a merger as a function of $z$ derived by dividing the number of expected mergers by the number of clusters present. To calculate the total amount of $r$-process material synthesized in these clusters we need to combine the rate calculations presented here with an estimate of the mass production rate per event. It is to this issue that we now turn our attention.

\section{3. $R$-PROCESS SYNTHESIS IN MERGING BINARIES}

The physical conditions that characterized the decompressed ejecta from compact binary mergers (Lattimer \& Schramm 1974) are compatible with the assembly of an $r$ process pattern that is generally consistent with solar system abundances (Freiburghaus et al. 1999). The most recent numerical studies of circular (i.e., zero eccentricity) compact binary mergers (e.g., Bauswein et al. 2013; Hotokezaka

\footnotetext{
${ }^{10}$ If, instead of equal mass, we assume the binary companion mass is three times the NS mass $\left(4.2 M_{\odot}\right.$, or a turnoff age of $\left.\approx 300 \mathrm{Myr}\right)$ then the effective exchange cross section is reduced (Heggie et al. 1996), but if an exchange occurs, the resultant binary is typically tighter by a factor of $a_{f} \approx m_{\text {cap }} / m_{\text {ej }} a_{0}$. Both of these effects play a role in determining the cross section for rapidly merging NS binaries. Under these assumptions $R_{\text {min }}^{3 \text {-body }}=3.0 \times 10^{5} \mathrm{~km}$, a factor of $\approx 5$ smaller than Equation (5).

${ }^{11}$ Essentially, with this choice we are providing the encounter rate at $z>6$ $(t<1 \mathrm{Gyr})$ and we note that compact binaries will survive cluster tidal dissolution if that happens before the merger.
}

et al. 2013) shows that they eject about $10^{-3}-10^{-2} M_{\odot}$ of $r$ process material (Lee \& Ramirez-Ruiz 2007; Faber \& Rasio 2012).

In contrast to these circular mergers, eccentric mergers (i.e., with finite eccentricity) can result in tidal tails that will synthesize significantly larger masses of $r$-process rich material (Lee et al. 2010; Rosswog et al. 2013). But in order for them to offer a consequential enhancement to standard circular mergers, the synthesized $r$-process mass should be higher by a factor of about $R_{\min }^{\mathrm{gw}} / R_{\min }^{\text {tidal }}$ when compared to circular $r$ process production. This requirement can be written as

$$
\frac{M_{r-p}^{\mathrm{tidal}}}{M_{r-p}^{\mathrm{gw}}} \lesssim \frac{R_{\min }^{\mathrm{gw}}}{R_{\min }^{\mathrm{tidal}}}=115.9\left(\frac{\sigma_{c}}{20 \mathrm{~km} \mathrm{~s}^{-1}}\right)^{-4 / 7}
$$

For the two binary members to come together and eventually merge, they must lose orbital angular momentum and energy. This can be achieved through gravitational wave emission, or mass ejection, or a combination of both. The tidal interaction provides an efficient mechanism for merging by ejecting a small amount of mass through the formation of tidal tails that carry a great deal of angular momentum to a large radius. In the case of a tidal capture, it is easier to dynamically unbind matter to infinity and, as a result, the amount of mass ejected is larger than in a merger by about one order of magnitude. In the case displayed in Figure $4, M_{r-p}^{\text {tidal }} / M_{r-p}^{\mathrm{gw}} \approx 4$ with $M_{r-p}^{\mathrm{gw}}=0.19 M_{\odot}$. Since $\quad M_{r-p}^{\text {tidal }} / M_{r-p}^{\mathrm{gw}} \ll R_{\min }^{\mathrm{gw}} / R_{\min }^{\text {tidal }}<R_{\min }^{3 \text {-body }} / R_{\min }^{\text {tidal }}$, circular binary mergers arising from binary exchange ${ }^{12}$ and $\mathrm{GW}$ capture should dominate the $r$-process mass production in high- $z$ stellar clusters. In what follows we thus neglect the contribution of eccentric mergers.

In Figure 4, the final elemental abundances in the tidal tails of a merging NS binary from Roberts et al. (2011) are compared with those of an eccentric binary resulting from the tidal capture of a NS by a black hole, both using the FPS equation of state (Shibata et al. 2005). The model results are also compared with observed elemental abundances of the CEMP-r star CS 22892-052 (Sneden et al. 2003). The evolution of the nuclear composition in the tails is followed using the nuclear reaction network described in Roberts et al. (2011), but symmetric fission has been assumed. All models assumed $Y_{e}=0.1$.

The models lack light $r$-process elements due to the extremely neutron-rich conditions encountered in the tidal ejecta and because we do not follow the disk formed after merger (Just et al. 2014). For the second and third $r$-process peaks, our abundance distribution is reasonably consistent with that seen in CEMP- $r$ stars (Sneden et al. 2008). The origin of the remaining discrepancy is likely due to the unsettled nuclear physics employed, in particular to the uncertainties in the fission rates and daughter distributions, and because $Y_{e}$ is unconstrained by the merger models used in this work.

\section{DISCUSSION}

Having established the amount of mass of $r$-process material produced by both mergers and tidal captures we can now proceed to determine the relevance of dynamically assembled binaries in enriching CEMP- $r$ stars with Eu. Figure 5 shows the cumulative mass of $r$-process material as a function of $z$

\footnotetext{
12 In the binary channel, only $\approx 1 / 108$ of the exchanges will result in eccentric merging binaries (Samsing et al. 2014).
} 

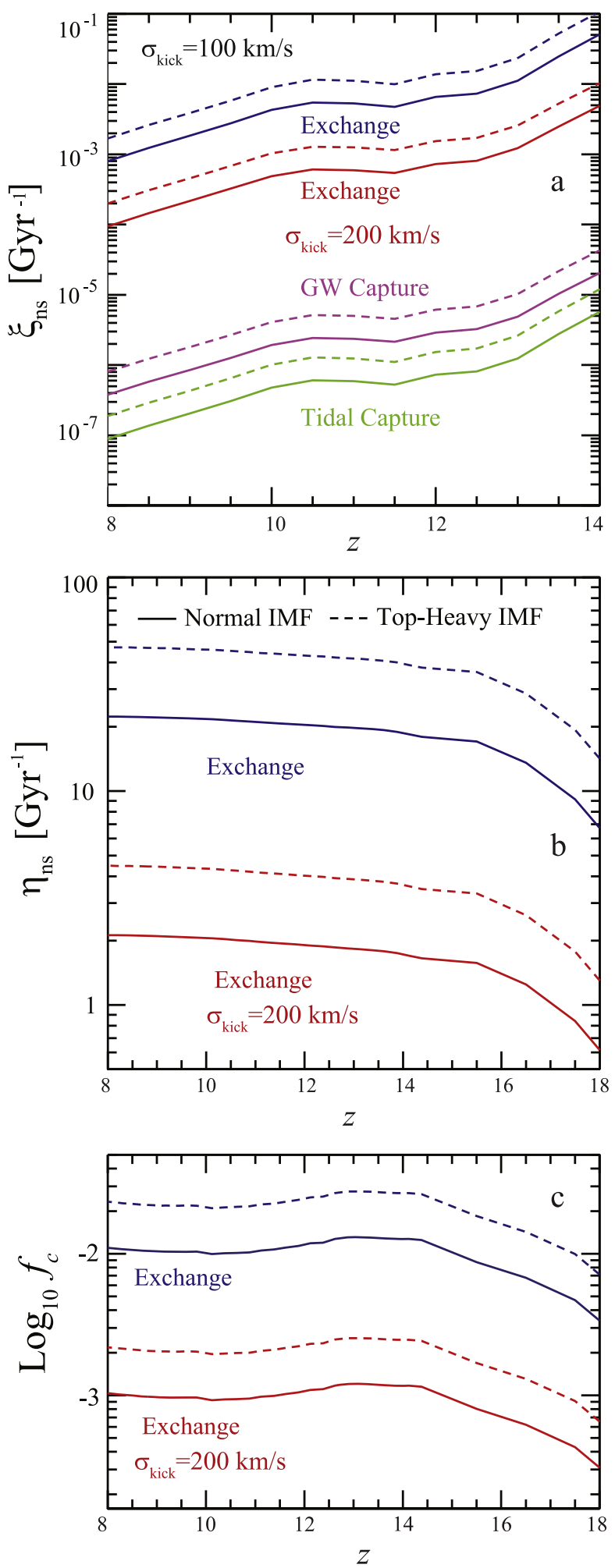

Figure 3. Formation rate of compact binaries in the early universe. Panel (a): the binary assembly rate $\xi_{\text {ns }}$ for individual clusters as a function of their formation redshift. The rate is higher for the clusters formed at higher redshift, because they have higher concentrations. Panel (b): the integrated event rates $\eta_{\mathrm{ns}}$ for dynamically formed binaries as a function of $z$. For this we have used the cluster formation history plotted in Figure 1 and then assumed that each cluster is active in producing collisions for $0.5 \mathrm{Gyr}$ at the rate set by its formation redshift. In addition, at $z \geqslant 14$ we have assumed a constant encounter rate as the Devecchi \& Volonteri (2009) model does not extend to higher $z$. This is a conservative assumption as the encounter rate increases with redshift. Panel (c): the fraction of star clusters $f_{c}$ that experienced a merger as a function of redshift.

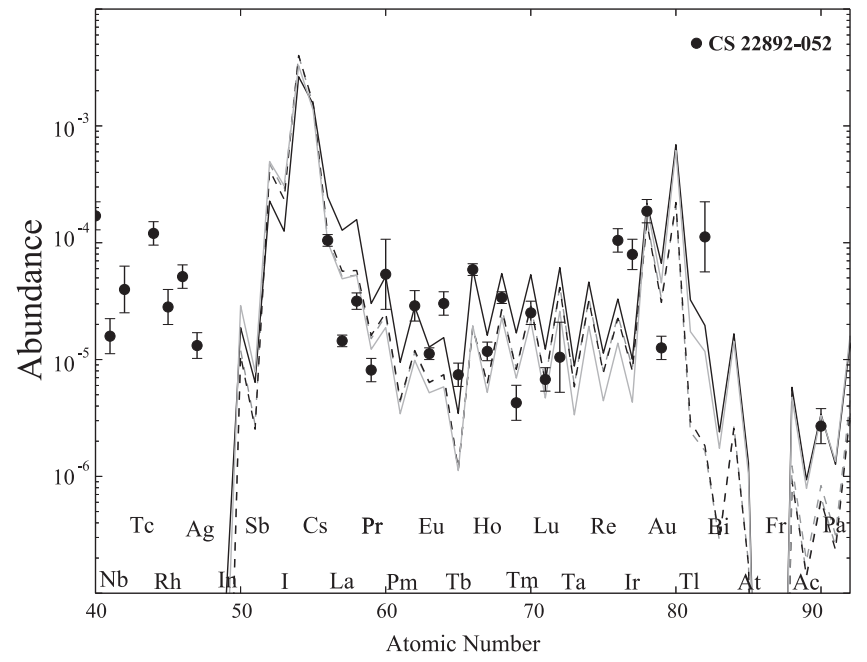

Figure 4. Abundance patterns calculated for a tidal encounter (solid lines) and a binary merger (dashed lines) compared with the most recent abundance data for the CEMP- $r$ star CS 22892-052 for which $[\mathrm{Fe} / \mathrm{H}]=-3.1$. The merging neutron star binary has a mass ratio $q=0.88$ while the eccentric binary is assembled by the tidal capture of a neutron star by a black hole with $R_{\min }=2.3 R_{\mathrm{ns}}$. The dashed lines show the abundances of two particle trajectories in the merging binary as calculated in Roberts et al. (2011) while the solid lines correspond to the abundances of two particle trajectories in the tidal capture encounter, which have been selected to define the extent of the abundance variation in the calculations. The abundance is defined as the number of a particular element per baryon and the CS 22892-052 data have been rescaled for the best fit to the network calculations.

synthesized in binary mergers, as calculated from the results shown in Figure 3. Typical model uncertainties of $5 \times 10^{-3} M_{\odot} \lesssim M_{r-p}^{\mathrm{gw}} \lesssim 2 \times 10^{-2} M_{\odot}$ are shown as the blue shaded region. For comparison we have plotted the contribution of Type II SN, calculated assuming a mass production rate of $M_{r-p}^{\mathrm{sn}}=10^{-5} M_{\odot}$, which is the average mass required per event in order for core collapse to be solely responsible for the $r$-process in the Milky Way (Cowan \& Thielemann 2004). In accordance with the higher level of scatter of $[\mathrm{Eu} / \mathrm{Fe}]$ in relation to $[\alpha / \mathrm{Fe}]$, which is most pronounced at values below $[\mathrm{Fe} / \mathrm{H}] \approx$ -2.0 , we have assumed that only a $5 \%$ of all Type II that produce $\alpha$-elements also yield $r$-process elements (Fields et al. 2002).

Figure 5 shows that in high- $z$ clusters, albeit with large uncertainties, dynamically assembled mergers could be potentially as important as Type II SNe. Because they happen only in a few clusters (Figure 3), we expect high concentrations of $r$ processes in locations where a binary merger happens compared to a case where Type-II SNe are assumed to be the main source of the $r$-process. We estimate that NS-NS merger blast waves do not carry enough energy and momentum to escape their host minihalo. However, the short halo assembly time at high- $z$ implies that there is significant mixing because of halo mergers, leading to an enhancement of $r$-process by a factor $\gtrsim 10$ (for $f_{c} \sim 10^{-2}$ ) in a few percent of the stars formed at $z \gtrsim 6$. This can help explain the large star-to-star bulk scatter in the concentrations of heavy elements with respect to the lighter metals in CEMP- $r$ stars (Sneden et al. 2008).

Studies of element abundances in the oldest and most $\mathrm{Fe}$ deficient stars in our Galaxy have concluded that, because NS binary timescales are long ( $\gtrsim 100 \mathrm{Myr})$, only SNe could have contributed to $r$-process synthesis at the earliest times (e.g., Cowan \& Thielemann 2004). However, recent hydrodynamical 


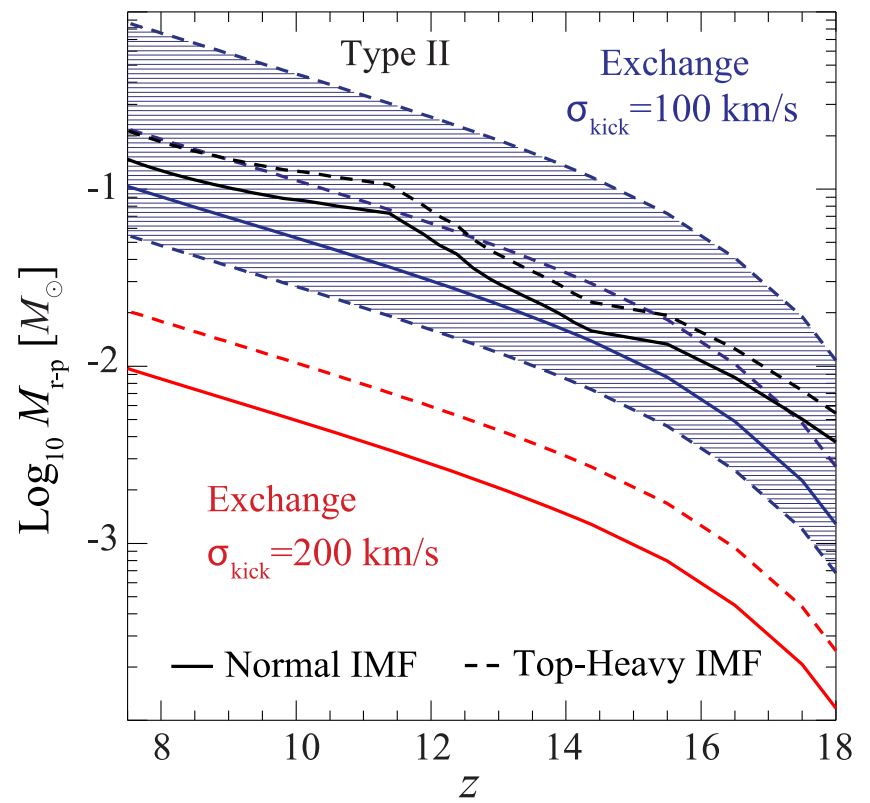

Figure 5. Predicted production of $r$-process elements from compact binaries in high- $z$ clusters. The red lines show production by Type II, while the blue lines show production by binary mergers. We assume that the mass of $r$-process material produced per events is $M_{r-p}^{\mathrm{sn}}=10^{-5} M_{\odot}$ and $5 \times 10^{-3} M_{\odot} \lesssim$ $M_{r-p}^{\mathrm{gw}} \lesssim 2 \times 10^{-2} M_{\odot}$ (blue shaded region), for Type II and compact binary mergers respectively. Also, we assume that only $5 \%$ of all Type II events produce $r$-process consistent with the view that only a small fraction of the massive stars in the early universe that produce $\alpha$-elements also yield $r$-process elements (Fields et al. 2002).

simulation studies have shown that assuming a relatively small time interval between the formation of the Galaxy and appearance of stars with $[\mathrm{Fe} / \mathrm{H}] \leqslant-3$ is not accurate (Shen et al. 2014; van de Voort et al. 2014) and that in fact NS mergers offer a favorable alternative to Type II SNe as the $r$ process site. For CEMP- $r$ stars, which are considered the chemical descendants of Population III stars (Umeda \& Nomoto 2003; Cooke \& Madau 2014), any $r$-process enhancement must happen on a timescale shorter than that of pollution from a second generation of stars. Dynamically assembled binaries resulting in a short-lived merger can fit these requirements better than the standard compact mergers, whose formation timescale is determined by binary stellar evolution and is estimated to be $\gtrsim 100$ Myrs (Fryer et al. 1999; Kalogera et al. 2001; Belczynski et al. 2006; Behroozi et al. 2014). Their production rate in high- $z$ clusters, as calculated in Section 2.2, could attain a sufficiently high value for compact binary mergers to still be a potentially viable source of heavy $r$-process material in CEMP- $r$ stars.

We thank S. Shen, R. Cooke, E. Kirby, C. Miller, M. Rees, and S. Rosswog for insightful discussions as well as the editor and referees for insightful suggestions. We acknowledge financial support from the Packard Foundation, NSF
(AST0847563), UCMEXUS (CN-12-578), and the Einstein Fellowship (L.R.).

\section{REFERENCES}

Argast, D., Samland, M., Thielemann, F.-K., \& Qian, Y.-Z. 2004, A\&A, 416, 997

Bauswein, A., Goriely, S., \& Janka, H.-T. 2013, ApJ, 773, 78

Behroozi, P. S., Ramirez-Ruiz, E., \& Fryer, C. L. 2014, arXiv:1401.7986

Belczynski, K., Perna, R., Bulik, T., et al. 2006, ApJ, 648, 1110

Cooke, R., \& Madau, P. 2014, ApJ, 791, 116

Cowan, J. J., \& Thielemann, F.-K. 2004, PhT, 57, 47

Devecchi, B., \& Volonteri, M. 2009, ApJ, 694, 302

Faber, J. A., \& Rasio, F. A. 2012, LRR, 15, 8

Fields, B. D., Truran, J. W., \& Cowan, J. J. 2002, ApJ, 575, 845

Freiburghaus, C., Rosswog, S., \& Thielemann, F.-K. 1999, ApJL, 525, L121

Fryer, C. L., Woosley, S. E., \& Hartmann, D. H. 1999, ApJ, 526, 152

Grindlay, J., Portegies Zwart, S., \& McMillan, S. 2006, NatPh, 2, 116

Grossman, D., Korobkin, O., Rosswog, S., \& Piran, T. 2014, MNRAS, 439, 757

Heggie, D. C., Hut, P., \& McMillan, S. L. W. 1996, ApJ, 467, 359

Hotokezaka, K., Kiuchi, K., Kyutoku, K., et al. 2013, PhRvD, 87, 024001

Just, O., Bauswein, A., Ardevol Pulpillo, R., Goriely, S., \& Janka, H.-T. 2014, arXiv: 1406.2687

Kalogera, V., Narayan, R., Spergel, D. N., \& Taylor, J. H. 2001, ApJ, 556, 340

Kim, S. S., \& Lee, H. M. 1999, A\&A, 347, 123

King, I. R. 1966, AJ, 71, 64

Kroupa, P. 2001, MNRAS, 322, 221

Krumholz, M. R., Klein, R. I., McKee, C. F., Offner, S. R., \& Cunningham, A. J. 2009, Sci, 323, 754

Hurley, J. R., Pols, O. R., \& Tout, C. A. 2000, MNRAS, 315, 543

Lattimer, J. M., \& Schramm, D. N. 1974, ApJL, 192, L145

Lee, H. M., \& Ostriker, J. P. 1986, ApJ, 310, 176

Lee, M. H. 1993, ApJ, 418, 147

Lee, W. H., \& Ramirez-Ruiz, E. 2007, NJPh, 9, 17

Lee, W. H., Ramirez-Ruiz, E., \& van de Ven, G. 2010, ApJ, 720, 953

Masseron, T., Johnson, J. A., Plez, B., et al. 2010, A\&A, 509, AA93

Matteucci, F., Romano, D., Arcones, A., Korobkin, O., \& Rosswog, S. 2014, MNRAS, 438, 2177

Metzger, B. D., Martinez-Pinedo, G., Darbha, S., et al. 2010, MNRAS, 406, 2650

Pfahl, E., Rappaport, S., \& Podsiadlowski, P. 2002, ApJ, 573, 283

Qian, Y.-Z., \& Woosley, S. E. 1996, ApJ, 471, 331

Roberts, L. F., Kasen, D., Lee, W. H., \& Ramirez-Ruiz, E. 2011, ApJL, 736, L21

Rosswog, S., Piran, T., \& Nakar, E. 2013, MNRAS, 430, 2585

Samsing, J., MacLeod, M., \& Ramirez-Ruiz, E. 2014, ApJ, 784, 71

Shen, S., Cooke, R. J., Ramirez-Ruiz, E., et al. 2014, arXiv:1407.3796

Sigurdsson, S., \& Phinney, E. S. 1993, ApJ, 415, 631

Shibata, M., Taniguchi, K., \& Uryu, K. 2005, PhRvD, 71, 084021

Sneden, C., Cowan, J. J., \& Gallino, R. 2008, ARA\&A, 46, 241

Sneden, C., Cowan, J. J., Lawler, J. E., et al. 2003, ApJ, 591, 936

Starkenburg, E., Shetrone, M. D., McConnachie, A. W., \& Venn, K. A. 2014 MNRAS, 441, 1217

Takahashi, K., Witti, J., \& Janka, H.-T. 1994, A\&A, 286, 857

Trenti, M., \& Shull, J. M. 2010, ApJ, 712, 435

Trenti, M., \& Stiavelli, M. 2009, ApJ, 694, 879

Trenti, M., Stiavelli, M., \& Shull, J. M. 2009, ApJ, 700, 1672

Trenti, M., Vesperini, E., \& Pasquato, M. 2010, ApJ, 708, 1598

Truran, J. W., Cowan, J. J., Pilachowski, C. A., \& Sneden, C. 2002, PASP, 114,1293

Umeda, H., \& Nomoto, K. 2003, Natur, 422, 871

van de Voort, F., Quataert, E., Hopkins, P. F., Keres, D., \& Faucher-Giguere, C.-A. 2014, arXiv:1407.7039

Wise, J. H., Turk, M. J., Norman, M. L., \& Abel, T. 2012, ApJ, 745, 50

Woosley, S. E., Wilson, J. R., Mathews, G. J., Hoffman, R. D., \& Meyer, B. S. 1994, ApJ, 433, 229 